\title{
Inter-channel Interference in Non-linear Frequency-division Multiplexed Networks on Fibre Links with Lumped Amplification
}

\author{
Xianhe Yangzhang, Domaniç Lavery and Polina Bayvel \\ University College London, Torrington Place, London WC1E 7JE, United Kingdom
}

There has been some interest in the Non-linear Frequency-Division Multiplexing (NFDM) in optical fibre communication systems, because it promises interference-free or weak-interference between channels in an optical routed network [1]. NFDM scheme uses Non-linear Fourier Transform (NFT) to bring a time-domain signal into the non-linear frequency domain (NFD), where the spectra evolve in a linear manner during signal propagation in the fibre channel. The successful application of NFT relies heavily on the "channel's integrability" that is only fulfilled by an ideal distribution-Raman amplification. However, most of the optical fibre links are amplified by Erbium-doped fibre amplifiers (EDFAs). The impact of the non-integrability in NFDM networks is unclear. In such a network, one key device is the Non-linear Add-drop Multiplexer (NADM) that adds or drops channels in the NFD [2]. Simulating such device that processes many channels simultaneously is still difficult due to a high complexity and inaccuracy of the current INFT-NFT algorithm. To get around this difficulty, we adopt a different approach to estimate the inter-channel interference (ICI) in NFDM networks.

The optical fibre channel model of concern is a multi-span dual-polarisation (DP) dispersion unmanaged fibre link, which can be described by the Manakov equation as in (1)

$$
\frac{\partial \vec{Q}}{\partial z}+\frac{\alpha}{2} \vec{Q}+\frac{j \beta_{2}}{2} \frac{\partial^{2} \vec{Q}}{\partial t^{2}}-j \frac{8}{9} \gamma \vec{Q}\|\vec{Q}\|^{2}=0, \quad \text { (1) } \quad \frac{\partial \overrightarrow{Q_{\mathrm{pa}}}}{\partial z}+\frac{j \beta_{2}}{2} \frac{\partial^{2} \overrightarrow{Q_{\mathrm{pa}}}}{\partial t^{2}}-j \frac{8}{9} \gamma_{a} \overrightarrow{Q_{\mathrm{pa}}}\left\|\overrightarrow{Q_{\mathrm{pa}}}\right\|^{2}=0
$$

where $\vec{Q}(t, z)=\left[Q_{1}(t, z) Q_{2}(t, z)\right]$ is the complex envelope of the DP-signal as a function of time $t$ and distance $z$ along the fibre. Other parameters are listed in the table in Fig. 1. To apply NFT, we approximate (1) with the socalled path-averaged Manakov equation as written in (2), where $\gamma_{a}=\gamma\left(1-e^{-\alpha L_{\mathrm{sp}}}\right) /\left(\alpha L_{\mathrm{sp}}\right)$. After each EDFA at $z=M L_{\mathrm{sp}}, M=1,2, \ldots, N_{\mathrm{sp}}, \overrightarrow{Q_{\mathrm{pa}}}(t, z)$ approximates $\vec{Q}(t, z)$ with small error. We first clarify the concepts of different digital back-propagation (DBP) schemes, as DBP is the main tool in our estimation. The single-channel DBP (SCDBP) refers to the process of filtering the channel of interest (COI) and solving (1) to recover $\vec{Q}(0, t)$ (input) from the filtered boundary condition $\vec{Q}(z, t)$ (output), using SSFM with fine step size $(0.1 \mathrm{~km})$. The EDFAs are replaced with attenuators of the opposite gain. The path-averaged DBP (PA-DBP) refers to the process of solving (2) to recover $\vec{Q}(0, t)$ from the boundary condition $\vec{Q}(z, t)$, using SSFM with fine step size $(0.1 \mathrm{~km})$. We compare two noiseless Wavelength-Division Multiplexed (WDM) systems illustrated in Fig. 1(a)(b) using DP 32-QAM Nyquist signal of $50 \mathrm{GHz}$ bandwidth with equalisation: 1) full-band PA-DBP followed by matched filter, 2) matched filter followed by SC-DBP. In single-channel scenario, if viewed as a equalisation scheme, the combination of NFT, back-rotation in the NFD, and INFT is somewhat equivalent to the PA-DBP. Therefore, we consider the residual distortion in the system of Fig. 1(a) as a rough estimate of the ICI in NFDM networks.
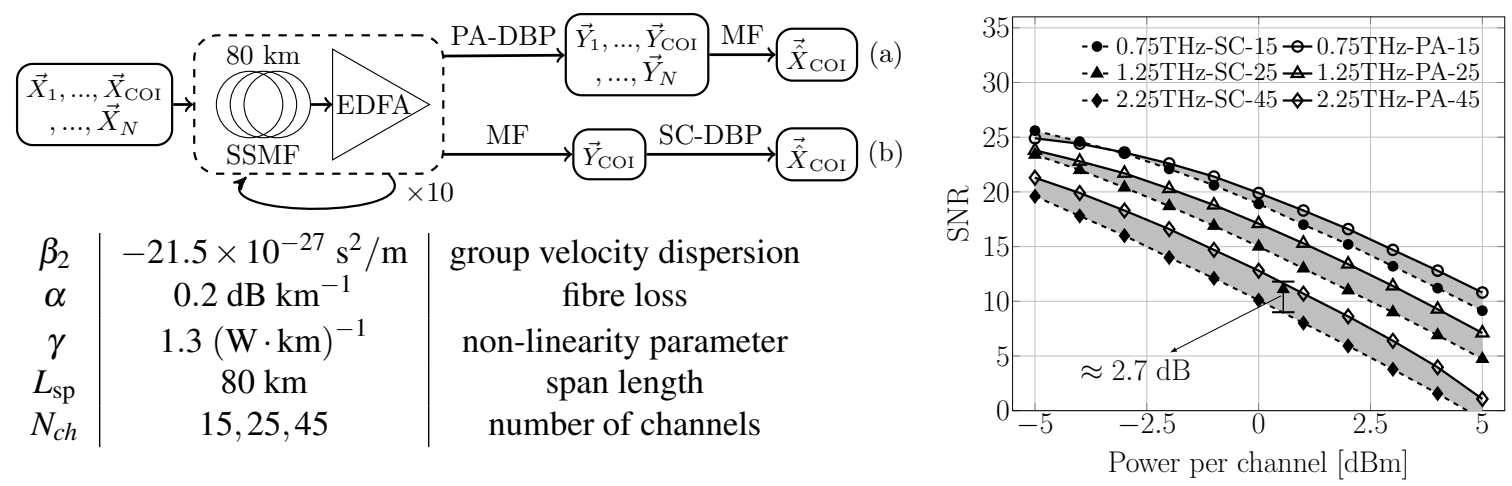

Fig. 1. (a)(b) Simulation diagram of noiseless non-integrable models. MF for matched filter. (c) Residual distortion of SCDBP (In the legend preceded by total bandwidth and followed by number of channels) and PA-DBP systems.

The estimated ICI in NFDM is weaker than ICI in WDM with SC-DBP when $N_{c h}$ is larger than 25.

\section{References}

1. M. I. Yousefi et al., "Linear and nonlinear frequency-division multiplexing," in ECOC, Sept 2016.

2. M. I. Yousefi et al., "Linear and nonlinear frequency-division multiplexing," arXiv:1603.04389v3, May 2016. 